\title{
Equilateral Triangles in Finite Metric Spaces
}

\author{
Vania Mascioni \\ Department of Mathematical Sciences \\ Ball State University \\ vdm@cs.bsu.edu
}

Submitted: Aug 2, 2003; Accepted: Feb 7, 2004; Published: Feb 27, 2004

MR Subject Classifications: 05C55, 05C12

\begin{abstract}
In the context of finite metric spaces with integer distances, we investigate the new Ramsey-type question of how many points can a space contain and yet be free of equilateral triangles. In particular, for finite metric spaces with distances in the set $\{1, \ldots, n\}$, the number $D_{n}$ is defined as the least number of points the space must contain in order to be sure that there will be an equilateral triangle in it. Several issues related to these numbers are studied, mostly focusing on low values of $n$. Apart from the trivial $D_{1}=3, D_{2}=6$, we prove that $D_{3}=12, D_{4}=33$ and $81 \leq D_{5} \leq 95$.
\end{abstract}

In classical combinatorial theory the following is a well-known, widely open problem: determine the minimal order of a complete graph such that when coloring the edges with $n$ colors (with $n \in \mathbb{N}$ fixed) we can find at least one monochromatic triangle. Such a smallest integer has been (among others) proved to exist by Ramsey [10] and is typically denoted by

$$
R_{n}[\underbrace{3,3, \ldots, 3}_{n \text { times }}]
$$

(since we won't consider any of the many variations of the problem, we will be using the simpler notation $R_{n}$ for short). People not acquainted with the theory are invariably surprised when learning that very little is known about $R_{n}$ (see [9] for Radziszowski's continuously updated survey of results): beyond the trivial cases $R_{1}=3, R_{2}=6$, the only known value is $R_{3}=17$, while for $R_{4}$ just the range $51 \leq R_{4} \leq 62$ has been established: the quality of the latter result should not be underestimated, since it took almost fifty years to improve the upper bound from 66 to 62 (a computer-free proof due to R.L. Kramer that $R_{4} \leq 62$ is more than 100 pages long $\left.[3,7]\right)$.

In this paper we will study a very much related, but technically different problem (to the best of our knowledge this problem appears to be new, which explains the lack of direct references). The distances between points of any finite metric space ("fms" for 
short) always belong to a finite set, and so the theory of fms reduces to when the distances are non-negative integers from, say, a set $S \subset \mathbb{N}$. Let us call such an fms an $S$-space. If an $S$-space $M$ is given, we may consider the points of $M$ as vertices of a complete graph, and the distances as colors applied to the edges. The difference, of course, is that distances must satisfy the triangle inequality, while in the classical Ramsey problem described above no such restriction is made on colors. In the following we will talk about metric spaces with distances in sets not containing 0: this slight abuse of language is only meant to simplify the discourse, since while 0 is a distance in every metric space, it only appears in the trivial expressions of the form $d(a, a)=0$ : in other words, by "distance" we will routinely mean "distance between different points."

Definition 1. For $S \subset \mathbb{N}$ we define $D[S]$ to be the smallest integer $m$ such that any finite metric space ( $=\mathrm{fms}$ ) consisting of $m$ points and with distances in the set $S$ must contain an equilateral triangle (i.e., three points $a, b, c$ with $d(a, b)=d(b, c)=d(c, a)$ ). For simplicity, we may drop the braces, as in $D[\{1,2,4\}]=: D[1,2,4]$, and we also define $D_{n}:=D[1,2, \ldots, n]$. Finally, let us call an fms eq-free if no three points in it form an equilateral triangle.

To summarize our main results on the low-index $D_{n} \mathrm{~s}$, and to put them in perspective compared to the known facts about the $R_{n} \mathrm{~s}$, we can look at the following table (exact references for all the results on $R_{n}$, are given in [9]):

\begin{tabular}{c|c|c}
$n$ & $D_{n}$ & $R_{n}$ \\
\hline 1 & 3 & 3 \\
2 & 6 & 6 \\
3 & 12 & 17 \\
4 & 33 & $51 \leq R_{4} \leq 62$ \\
5 & $81 \leq D_{5} \leq 95$ & $162 \leq R_{5} \leq 307$ \\
6 & $251 \leq D_{6} \leq 389$ & $538 \leq R_{6} \leq 1838$ \\
7 & $551 \leq D_{7} \leq 1659$ & $1682 \leq R_{7} \leq 12861$
\end{tabular}

Apart from the asymptotics discussed at the very end, essentially this paper is about proving the results listed in the $D_{n}$ column (proved below in Theorems 2, 11, 19, 22 and 23; see also the inequality in Theorem 21). We will complement these statements with uniqueness-type results for eq-free spaces with a maximal number of points (Theorems 4, 14, 15, 16 and 20). In particular, a good deal of work will be needed in order to obtain the fact that there only exist two non-isomorphic 32-point eq-free fms with distances in $\{1,2,3,4\}$ (see Theorem 20): despite the complications, though, the proof of this result is made a lot easier by the known results by Kalbfleisch and Stanton [6] (see also [8]) on the two possible 3 -colorings of the edges of $K_{16}$ with no monochromatic triangles. It thus appears that the theories of the $R_{n}$ and the $D_{n}$ numbers, though technically different, may end up helping each other.

Let us now start the investigation. First (as we restate below in Corollary 5 for easier reference), note that we always have $D_{n} \leq R_{n}$ : still, clearly the numbers $D_{n}$ are expected 
to be smaller than their $R_{n}$ counterparts except for the cases $n=1$ and $n=2$ : in fact, the study of $D_{1}$ and $D_{2}$ is just the same as the one of $R_{1}$ and $R_{2}$, and this simply because in any $\{1\}$ - or $\{1,2\}$-space any triangle is "legal." In these cases we are back to full equivalence between distances and colors and the problem is exactly the classical Ramsey problem. We thus have the following easy

Theorem 2. We have $D_{1}=3$, and $D_{2}=6$. More generally, let $1 \leq k \leq l(k, l \in \mathbb{N})$ :

$$
\begin{gathered}
D[k]=3 \\
D[k, l]= \begin{cases}5 \quad, & 2 k \geq l \\
6 \quad, & 2 k<l\end{cases}
\end{gathered}
$$

Proof. The only part we need to discuss is where the distance set $S=\{k, l\}$ satisfies the inequality $2 k<l$, and therefore triangles with sides of length $k, k, l$ are not allowed. First, it should be clear that $D[k, l] \leq D_{2}=6$. On the other hand, a four-point eq-free fms can easily be constructed: just label the points $a_{1}, a_{2}, a_{3}, a_{4}$ and define $d\left(a_{1}, a_{2}\right)=d\left(a_{3}, a_{4}\right)=$ $k$, with all the other pairs being at distance $l$. To the reader we leave the verification that no eq-free $\{k, l\}$-space can exist with five points.

As in the classical Ramsey case, things start getting dicey with $D_{3}$. The rest of the paper is dedicated to the study of the numbers $D_{n}$ and of some variations thereof, as defined in Definition 1. To get some concrete examples of eq-free fms out of the way, and to have them readily available later, we start by looking at a general idea to stay eq-free with increasing number of different distances:

Example 3. Let $M$ have $m:=3 \cdot 2^{n-1}-1$ points, and label them as $v_{1}, \ldots, v_{m}$. We can think of $M$ as the complete graph $K_{m}$ with vertex set $\mathbb{Z}_{m}$, and for $i, j \in\{1, \ldots, m\}$ define a "cyclic metric" by $d\left(v_{i}, v_{j}\right)=k$ iff the distance between $i-1$ and $j-1$ in $\mathbb{Z}_{m}$ is in $\left\{2^{k-1}, 2^{k-1}+1, \ldots, 2^{k}-1\right\}$. More explicitly, for every pair of indices $1 \leq i<j \leq m$ we define

$$
d\left(v_{i}, v_{j}\right):=\left\{\begin{array}{cll}
1 & : & j-i \in\{1, m-1\} \\
2 & : & j-i \in\{2,3, m-3, m-2\} \\
3 & : & j-i \in\{4,5,6,7, m-7, m-6, m-5, m-4\} \\
\ldots & : & \cdots \\
n-1 & : & j-i \in\left\{2^{n-2}, \ldots, 2^{n-1}-1, m-\left(2^{n-1}-1\right), \ldots, m-2^{n-2}\right\} \\
n & : & j-i \in\left\{2^{n-1}, 2^{n-1}+1, \ldots, 2^{n}-1\right\}
\end{array}\right.
$$

This gives (it's boring, but the reader is welcome to check!) an eq-free fms with $3 \cdot 2^{n-1}-1$ points and distances in $\{1,2, \ldots, n\}$, and thus shows that $D_{n} \geq 3 \cdot 2^{n-1}$. We will call this particular example $M_{n}$.

By a maximal eq-free $S$-space we mean an fms $M$ with the largest possible number of points among all $S$-spaces that are eq-free (by definition, we then have $|M|+1=D[S]$ ).

Note that $M_{1}$ (just two points at distance 1) and $M_{2}$ (five points arranged as vertices of a pentagon with edge length 1 , and with all the other distances being 2 ) are easily seen to 
be unique in the class of maximal eq-free fms with distances in the respective sets $\{1\}$ and $\{1,2\}$. Below we will prove that $D_{3}=12$ (Theorem 11), and so $M_{3}$ (with its 11 points) is a maximal eq-free $\{1,2,3\}$-space (with 11 points). The uniqueness of $M_{3}$ is a more delicate question than in the trivial cases of $M_{1}$ and $M_{2}$, and will be proved in Theorem 14. Similarly, we will prove the result $D_{4}=33$ in Theorem 19 and the corresponding uniqueness result (though in this case there will be two non-isomorphic maximal spaces) in Theorem 20. Of course, we will always understand that an isomorphism of fms is a distance-preserving, bijective function between two fms.

For the record, let us now state the uniqueness result for $M_{1}$ and $M_{2}$.

Theorem 4. $M_{1}$ (resp. $M_{2}$ ) are unique (up to isomorphism) among all maximal eq-free $\{1\}-$ (resp. $\{1,2\}-)$ spaces.

A perhaps more noteworthy consequence of Example 3 is the first inequality in the next corollary (the second one being a straight consequence of the definitions), but we will substantially improve on it in Theorem 21 further below:

Corollary $5 \cdot 3 \cdot 2^{n-1} \leq D_{n} \leq R_{n}$ (for all $n \geq 1$ ).

The purpose of the next "irregular" examples will be clear later, but we anticipate them now just so as not to interrupt the flow of later proofs.

Example 6. There exists a 10-point eq-free $\{1,2,4\}$-space: label the points in $M$ as $a_{1}, \ldots, a_{10}$, and assign them to five pairs $S_{i}:=\left\{a_{2 i-1}, a_{2 i}\right\}$. Define a metric by setting

$$
d(a, b):=\left\{\begin{array}{lll}
1 & : & a, b \in S_{j} \\
2 & : & a \in S_{j} \text { and } b \in S_{j+1} \\
4 & : & a \in S_{j} \text { and } b \in S_{j+2}
\end{array}\right.
$$

where we understand that the pairs $S_{j}$ are ordered cyclically (i.e., $S_{6}:=S_{1}, S_{7}=S_{2}$ ). To see that this indeed is a metric, note that the only triangles that might fail the triangle inequality in a $\{1,2,4\}$-space would be those with sides of length $1,1,4$ or those with sides of length 1,2,4. Now, if a triangle in $M$ has two sides of length 1 and 4 , by the definition it must contain two points $a, b$ in the same pair (wlog, $S_{1}$ ), and the third point $c$ in, say, $S_{3}$. This means that both $d(a, c)$ and $d(b, c)$ must be of length 4 , and so we see that the triangle must have sides of length $1,4,4$, which is perfectly legal.

An example of a 10-point eq-free $\{1,3,4\}$-space would be quite similar, with the only spot to change being the distance 2 in the definition of $d(\cdot, \cdot)$, which of course needs to be replaced by 3 . Note that in the case of $\{1,3,4\}$-spaces the only illegal triangles would be those with side lengths $1,1,3$ or $1,1,4$, but the same argument as given for the $\{1,2,4\}$ case shows that this example indeed gives an fms, too.

Example 7. To allow us to give "logical" and "constructive" names to more complex examples of fms, we now define the operation $\otimes$ : given two fms $E$ and $F$, define $E \otimes F$ to be the set obtained by replacing every point of $F$ by an isomorphic copy of $E$, and defining the distances between points of two different copies of $E$ as the distance between 
the points of $F$ they had replaced: depending on $E$ and $F$, this may not be an fms, but we will only use the construction to simplify notation, not to define a general "algebra" of fms. Also, given an fms $E$ and an integer $k \in \mathbb{N}$, we define the fms $k E$ to be space $E$ where all the original distances in $E$ have been multiplied times $k$. Similarly, we define $E+k$ to be the space $E$ where every distance has been augmented by $k$.

To put this in practice, the $\{1,2,4\}$-space defined in Example 6 would be called $M_{1} \otimes$ $2 M_{2}$. The similar $\{1,3,4\}$ space mentioned at the end of Example 6 would be called $M_{1} \otimes\left(M_{2}+2\right)$.

The following Lemma will prove useful when dealing with maximal eq-free fms:

Lemma 8. Let $M$ be a maximal eq-free S-space. Then for every point $a \in M$ and $\delta \in S \cap\{1,2\}$ there exists a point $b \in M$ such that $d(a, b)=\delta$.

Proof. Suppose not, that is, let $a \in M$ and $\delta \in S \cap\{1,2\}$ be such that for no $b \in M$ we have $d(a, b)=\delta$. Create a new point $\tilde{a}$ and add it to $M$ as follows: for $b \in M \backslash\{a\}$ define $d(\tilde{a}, b):=d(a, b)$, while we set $d(a, \tilde{a}):=\delta$. Notice that $\tilde{M}:=M \cup\{\tilde{a}\}$ is still metric (the only new triangles that may give us trouble are the "isosceles" ones with third side $a \tilde{a}$, and the latter can only have length 1 or 2 ). Also, it is immediate to see that $\tilde{M}$ is still eq-free and yet is larger than $M$, which contradicts $M$ 's maximality.

Definition 9. In the rest of this paper we will freely abuse graph-theoretic language as follows: given an fms $M$ where distance 1 is allowed, we will tacitly consider a graph whose vertices are the points of $M$, and whose edges connect exactly those pairs of points that are at distance 1 (we will call these points at distance 1 "neighbors"). Let's call this graph $G_{M}$ for the moment. If a cycle $C_{m}$ should be a subgraph of $G_{M}$, we will say that " $M$ contains a $C_{m}$ " (and often, if no confusion arises, we will just call the corresponding subspace of $M$ with the name $C_{m}$ ). Similarly, if $G_{M}$ contains a path $P_{n}$ as a subgraph, we will say " $M$ contains a $P_{n}$." In contrast to common graph-theoretic usage, we will use $P_{n}$ to denote a path with $n$ vertices (and not with $n$ edges, as usual), since our emphasis is on the number of points.

In order to make the language in the following proofs more bearable, we will adapt standard graph theory notation to our needs:

Definition 10. Let $M$ be an $S$-space. For $a \in M$ and $k \in S$, define the " $k$-neighborhood" of $a$ as $N_{k}(a):=\{b \in M: d(a, b)=k\}$. "Distance patterns" for a specific element of $M$ play a major role in the combinatorial arguments to follow. Assume that we have arranged the distances in $S$ in order, say, $k_{1}<k_{2}<\ldots<k_{r}$ : we say that $a \in M$ is of type

$$
\left[\left|N_{k_{1}}(a)\right|,\left|N_{k_{2}}(a)\right|, \ldots,\left|N_{k_{r}}(a)\right|\right]
$$

Given that

$$
\left|N_{k_{1}}(a)\right|+\left|N_{k_{2}}(a)\right|+\ldots+\left|N_{k_{r}}(a)\right|=|S|-1,
$$

under some assumptions only a few distance patterns will be available. 
We are now ready to prove our first deeper result, which should be compared to Greenwood and Gleason's $R_{3}=17$ :

Theorem 11. $D_{3}=D[1,2,3]=12$. In the case of other distance sets $S$ with three elements, we have

$$
D[1,2,4]=D[1,3,4]=11 .
$$

More generally, let $1 \leq k \leq l \leq m$ :

$$
D[k, l, m]= \begin{cases}11, & l \leq 2 k<m \text { and } k+l<m \\ 11, & 2 k<l \\ 12, & l \leq 2 k<m \text { and } m \leq k+l \\ 17, & m \leq 2 k\end{cases}
$$

Proof. To see that $D_{3}=12$, let $M$ be a maximal eq-free $\{1,2,3\}$-space, and fix $a \in M$. Since $M$ is eq-free, we must have $\left|N_{1}(a)\right| \leq 2,\left|N_{2}(a)\right| \leq 4$ and $\left|N_{3}(a)\right| \leq 5$ : this because $N_{1}(a)$ must be a $\{2\}$-space, $N_{2}(a)$ must be a $\{1,3\}$-space, and $N_{3}(a)$ must be a $\{1,2\}$-space (we will use this argument several times below, and it is simply based on the necessity to avoid equilateral triangles within an $N_{k}$ set) and because of the bounds set by Theorem 2 .

Overall, then, $|M| \leq 1+2+4+5=12$. Suppose $|M|=12$ (and thus that all $N_{k}$ sets have their largest possible size), and let $b \in M$ be such that $d(a, b)=3$. Since $\left|N_{3}(a)\right|=5,\left|N_{3}(b)\right|=5$, and since clearly $N_{3}(a) \cap N_{3}(b)=\emptyset$, there are only 2 points left in $M \backslash\left(N_{3}(a) \cup N_{3}(b)\right)$, and they both are at distance less than 3 from both $a$ and b. By Theorem 2, both $N_{3}(a)$ and $N_{3}(b)$ are maximal $\{1,2\}$-spaces, and thus must be isomorphic to $M_{2}$ (see Example 3 above), i.e., the points in each set must be arranged according to the same unique pattern: a pentagon where the sides have length 1 and any other distance is 2 .

Now, pick one of the two remaining points. Since it must be at distance 1 from two other points $\left(\left|N_{1}\right|=2\right.$ for all points in $\left.\left.M\right)\right)$, one of the two must belong to either $N_{3}(a)$ or $N_{3}(b)$, but this is impossible because it would imply that some point in $M$ is at distance 1 from 3 other points, a contradiction (these three points would then necessarily form an equilateral triangle).

So, $D_{3} \leq 12$. To see that there exists an eq-free $\{1,2,3\}$-space with 11 points, just consider the space $M_{3}$ defined in Example 3, and so our proof that $D_{3}=12$ is complete.

Time to prove that $D[1,2,4]=11$. Let us first show that $D[1,2,4] \leq 11$. By contradiction (and since $D[1,2,4] \leq 12$ because of $D_{3}=12$ : an eq-free $\{1,2,4\}$-space becomes an eq-free $\{1,2,3\}$-space just by redefining distance 4 to be 3$)$, assume that we have an eq-free, 11-point fms $M$ with distances in $\{1,2,4\}$.

It is not possible that every point in $M$ be at distance 1 from two other points: if this were the case, we could split the points of $M$ into disjoint cycles with side 1 . By the triangle inequality, the distances within each cycle could only be 1 and 2 (since distance 3 is unavailable), and so the cycles could have at most length 5 (since $D_{2}=D[1,2]=6$ by Theorem 2). Since they would also need to contain at least four points (a 3-cycle would 
be an equilateral triangle!), we would derive a contradiction as 11 cannot be written as a sum of $4 \mathrm{~s}$ and $5 \mathrm{~s}$.

It thus follows that $\left|N_{1}\right|=1$ for some point in $M$ (we must always have $N_{1} \neq \emptyset$ by the maximality of $M$ and Lemma 8): let $a \in M$ of type $[1,4,5]$ (the inequalities $\left|N_{2}\right| \leq 4$ and $\left|N_{4}\right| \leq 5$ hold everywhere for the same reason explained at the beginning of this proof). Call $b$ one of the 5 points in $N_{4}(a)$, and notice that these 5 points must be arranged as vertices of a pentagon of side 1, all the other internal distances being $2\left(N_{4}(a)\right.$ must be isomorphic to $M_{2}$ by Theorem 4). Now, we must have that $N_{4}(b)$ consists of four points: if not, it should contain five, but then $M$ would include two disjoint "pentagons" (as above), and the 11th point would have no points at distance 1, a contradiction with Lemma 8: this means that $b$ is of type $[2,4,4]$. So, we find exactly two points $\{x, y\}$ in $M \backslash\left(N_{4}(a) \cup N_{4}(b)\right)$. Since the only distances allowed are 1,2,4, the two points in $N_{1}(b)$ are already in $N_{4}(a)$, and neither $x$ nor $y$ is at distance 4 from $b$, we must have $d(x, b)=d(y, b)=2$. Calling $N_{1}(b)=\left\{b^{\prime}, b^{\prime \prime}\right\} \subset N_{4}(a)$, by the triangle inequality $x$ (and $y$ ) must be at distance 2 from both $b^{\prime}$ and $b^{\prime \prime}$, a contradiction, since $d\left(b^{\prime}, b^{\prime \prime}\right)=2$, and we would have an equilateral triangle in $M$. So, $D[1,2,4] \leq 11$ as we wanted. Finally, to see that $D[1,2,4]=11$, we only need an example of a 10-point, eq-free fms with distances in $\{1,2,4\}$, but this was given in Example 6 (and called $M_{1} \otimes 2 M_{2}$ according to the guidelines of Example 7).

Let us now check that $D[1,3,4]=11$. First note that $D[1,3,4] \geq 11$ follows from Example 6 (the space we called $M_{1} \otimes\left(M_{2}+2\right)$ is an eq-free, 10-point $\{1,3,4\}$-space). Pick $a \in M$. The points at distance 3 from $a$ must all have distances $\in\{1,4\}$ between each other, and so $\left|N_{3}(a)\right| \leq 4$ (by Theorem 2). Similarly, the points at distance 4 from $a$ must have all distances $\in\{1,3\}$ between each other, and so $\left|N_{4}(a)\right| \leq 4$. Since there cannot be more than one point at distance 1 from $a$ (distance 2 is not available here), this shows that $M$ can at most contain $1+1+4+4=10$ points, and thus $D[1,3,4]=11$.

Finally, the statement made for general distance sets $S=\{k, l, m\}$ looks harder but is now easy to verify, since the conditions imposed on $k, l, m$ are there to check which types of triangles are not allowed by the triangle inequality, and so the general $S$-space case falls back to either the previously considered cases, or else (when $2 k \geq m$, i.e., when every triangle is legal) we find ourselves in the classical Ramsey situation (where distances are equivalent to colors), and the stated result follows from the well-known $R_{3}=17$ (see $[5])$.

The next Lemma and Theorem will be used in the special cases $n=2$ and $n=3$, but since an inductive argument applies, we present them in full generality. See further below (Theorem 21) for an improvement on the technique.

Lemma 12. For all $n \geq 1$ we have

$$
D_{n+1} \geq 2 D_{n}-1 \geq D_{n}+\left|M_{n}\right|=D_{n}+3 \cdot 2^{n-1}-1 .
$$


Proof. Let $M$ be a maximal eq-free $\{1, \ldots, n\}$-space (with $|M|=D_{n}-1$ ). Build a $\{1, \ldots, n+1\}$-space $\hat{M}$ by letting $\hat{M}=M \otimes(n+1) M_{1}$ (i.e., we take the disjoint union of two copies of $M$, and define the distance between any point in one copy and any point of the other to be $=n+1)$. Clearly, $\hat{M}$ is eq-free and so

$$
|\hat{M}|=|M|+|M|=2\left(D_{n}-1\right) \leq D_{n+1}-1
$$

and the Lemma is proved.

Theorem 13. Suppose an eq-free $\{1, \ldots, n+1\}$-space $M(n \geq 2)$ contains an isomorphic copy of $M_{n}$ (just call the copy $M_{n}$, to keep things simple). Then, for every c $\in M \backslash M_{n}$ there exist at least $2^{n}-1$ points in $M_{n}$ at distance $n+1$ from c (that is, $\left|N_{n+1}(c) \cap M_{n}\right| \geq 2^{n}-1$ ). Consequently, under our hypothesis we must have $|M|<D_{n}+\left|M_{n}\right|$ and, if $n=2$ or $n=3$, $M$ cannot be maximal.

Proof. We first prove that if $c \in M \backslash M_{n}$ then there must be some $a \in M_{n}$ with $d(c, a)=n$. If not, by the triangle inequality either all points of $M_{n}$ must be at distance $n+1$ from $c$ (in which case the first part of the Theorem is proved), or else all points of $M_{n}$ are at distance $\leq n-1$ from $c$. Let $m$ be the largest distance $\in\{2,3, \ldots, n-1\}$ from $c$ to any point in $M_{n}$, and let this point be called $a$. Starting a count from $a$ and around $M_{n}$ (as usual we can visualize the points of $M_{n}$ as the vertices of a regular polygon with $3 \cdot 2^{n-1}-1$ sides and side length 1 ), the first point we meet that is at distance $m$ from $a$ is the $2^{m-1}$-th (by the definition of $M_{n}$ 's metric), and it is also the first of $2^{m-1}$ points that are all at distance $m$ from $a$. Clearly, none of these points can be at distance $m$ from $c$. Let $K$ be an interval of maximal length in $M_{n}$, all of whose points are at distance $\leq m-1$ from $c$. By what we just said, $|K| \geq 2^{m-1}$, but we can say more. By construction, the two points just outside $K$ must be both at distance $m$ from $c$. Call one of them $e$. Counting from $e$ in the direction of $K$ we find a first point at distance $m$ from $e$ after $2^{m-1}$ steps (which land still inside $K$ ), and then there must follow $2^{m-1}-1$ more points at distance $m$ from $e$. We deduce then $|K| \geq\left(2^{m-1}-1\right)+2^{m-1}=2^{m}-1$. We can now repeat this argument inductively by focusing on $K$, whose endpoints, by construction, must be both at distance $m-1$ from $c$. We would define $K^{\prime}$ to be an interval $\subset K$ of maximal length such that all of its points be at distance $<m-1$ from $c$, and so on. Eventually, by complete induction we will be able to exhibit an interval containing at least $2^{3}-1$ points and such that all of its elements are at distance 2 from $c$, but this is impossible.

So, let $a \in M_{n}$ be such that $d(c, a)=n$. By the definition of $M_{n}$ there is an "interval" $I$ of $2^{n-1}$ points in $M_{n}$ "opposite" $a$ such that $d(a, b)=n$ for all $b \in I$. This means that $d(c, b) \neq n$ for all $b \in I$. Let $J \supset I$ be such that $J$ is a maximal set of consecutive points of $M_{n}$ with the property that none of them is at distance $n$ from $c$. The neighbor $e$ of one of the endpoints of $J$ must be at distance $n$ from $c$. Counting from $e$ in the direction of $J$, we find that the first point at distance $n$ from $e$ occurs after $2^{n-1}$ steps: that is, the $2^{n-1}$-th element of $J$ (from e's end) is the first of $2^{n-1}$ points that are at distance $n$ from $e$, and therefore cannot be at distance $n$ from $c$. This means that $J$ must actually contain at least $\left(2^{n-1}-1\right)+2^{n-1}=2^{n}-1$ points, and none of them can be at distance $n$ from $c$. So, by the triangle inequality there are now two options: either all the points 
in $J$ are at distance $n+1$ from $c$ (and in this case we are done), or else they are all at distance in $\{2,3, \ldots, n-1\}$. The second option is however impossible (it could be seen as the starting point of the argument that got us a contradiction in the first part of this proof).

To verify the second part of the Theorem, let $b, c \in M \backslash M_{n}$. By the first part, there exist at least $2^{n}-1$ points in $M_{n}$ at distance $n+1$ from $b$, and the same (with possibly different points) applies to $c$. Now, these two sets of points must overlap, or else $M_{n}$ would need to have at least $2\left(2^{n}-1\right)=2^{n+1}-2$ points, but this is impossible since $\left|M_{n}\right|=2^{n}+2^{n-1}$. If we now pick a point $a \in M_{n}$ from the intersection, it must be at distance $n+1$ from both $b$ and $c$, that is, $d(b, c) \leq n$. Hence, $M \backslash M_{n}$ is an eq-free $\{1, \ldots, n\}$-space, and so $\left|M \backslash M_{n}\right|<D_{n}$. So, we have the inequality

$$
|M|=\left|M \backslash M_{n}\right|+\left|M_{n}\right|<D_{n}+\left|M_{n}\right| \leq D_{n+1},
$$

where the last inequality follows from Lemma 12. If in addition we have $n=2$ or $n=3$, then because of $D_{n}=\left|M_{n}\right|+1$ (an identity verified in Theorems 2 and 11) we could deduce that $|M| \leq 2\left|M_{n}\right|$, and since $2\left|M_{n}\right|<\left|M_{n+1}\right|$ (by just one!) and $\left|M_{n+1}\right|<D_{n+1}$, $M$ cannot be maximal.

The following result settles a similar question to the uniqueness of $M_{1}$ and $M_{2}$ (see Theorem 4) among all maximal eq-free $\{1\}$ - (resp. $\{1,2\}$-) spaces. It will all be worth the effort of going through the following streak of uniqueness theorems, though (Theorems 14, 15 and 16), since they will be an essential tool to tackle the issue of finding out more about $D_{4}$ (see Theorem 19).

Theorem 14. Up to isomorphism, $M_{3}$ is the only maximal eq-free $\{1,2,3\}$-space.

Proof. Let $M$ be an 11-point eq-free fms. The only possible types of points in $M$ are easily seen to be $[1,4,5],[2,4,4]$ and $[2,3,5]$ (by Lemma $8,\left|N_{1}\right| \in\{1,2\}$ everywhere and Theorem 2 implies that the inequalities $\left|N_{2}\right| \leq 4$ and $\left|N_{3}\right| \leq 5$ must hold at every point, so the identity $\left|N_{1}\right|+\left|N_{2}\right|+\left|N_{3}\right|=10$ does the rest).

However, no point of $M$ can be of type [*,*,5], or else $M$ would contain $M_{2}$, and by Theorem 13 we would have the contradiction $|M| \leq 10$. So, we immediately deduce that all the points of $M$ are of the same type $[2,4,4]$. Type $[2, *, *]$ for all the points means that if we regard $M$ as a graph with edges exactly where the distance between two vertices is 1 , then $M$ is a disjoint union of cycles, and these cycles must have length at least 4 (there was a similar argument in the proof of Theorem 11).

Claim: $M$ must be a unique cycle $\left(C_{11}\right)$. Since the only ways to add up to 11 with summands at least 4 are $4+7$ and $5+6$, we need to prove that a split of $M$ into two cycles contradicts our hypotheses. On one hand, note that a cycle $C_{6}$ is never possible in an eq-free space, since the triangle formed by every other point in such a cycle would be an equilateral triangle of side 2 . So, assume (by contradiction) that $M$ is a union of a cycle of length 4 and one of length 7 . Let $a \in M$ belong to the cycle of length 4 . This means that only one point (call it $b$ ) on this cycle is in $N_{2}(a)$, while the other three elements of $N_{2}(a)$ must belong to the other cycle $\left(\left|N_{2}(a)\right|=4\right.$ as we established that all points in $M$ 
are of type $[2,4,4])$. Since $\left|N_{2}(a)\right|=4$ and $N_{2}(a)$ is an eq-free $\{1,3\}$-space, by Theorem 2 it is a maximal eq-free $\{1,3\}$-space, which requires that $b$ must be at distance 1 from one of the other points in $N_{2}(a)$ : this, however, is impossible since no two points belonging to different cycles may be at distance 1 (or else $M$ could not be eq-free): the claim is thus proved.

We can now safely assume that $M$ is a $C_{11}$, that is, $d\left(a_{1}, a_{2}\right)=d\left(a_{2}, a_{3}\right)=\ldots=$ $d\left(a_{11}, a_{1}\right)=1$. If we could show that (applying cyclic permutations on the indices) $N_{2}\left(a_{1}\right)=\left\{a_{3}, a_{4}, a_{9}, a_{10}\right\}$, everywhere in $M$, we would have that $M$ is isomorphic to $M_{3}$. Therefore, we will look for a contradiction assuming that $a_{1}$ (say) does not have this property. In any case, note that we must always have $a_{3}, a_{10} \in N_{2}\left(a_{1}\right)$. What follows is the first of many arguments in the rest of this paper where the reader would probably find it easier to follow by means of a sketch or two.

Case I: $a_{4}, a_{9} \notin N_{2}\left(a_{1}\right)$ : In this situation we have $d\left(a_{1}, a_{4}\right)=d\left(a_{1}, a_{9}\right)=3$. We must then have $d\left(a_{1}, a_{5}\right)=3$ (or else $a_{1} a_{3} a_{5}$ would be equilateral) and $d\left(a_{1}, a_{8}\right)=3$ (or else $a_{1} a_{8} a_{10}$ would be equilateral). This leaves us with $d\left(a_{1}, a_{6}\right)=d\left(a_{1}, a_{7}\right)=2$. Now, we check that triangle $a_{3} a_{6} a_{10}$ is equilateral: in fact, $d\left(a_{3}, a_{6}\right)=3$ (since $d\left(a_{1}, a_{3}\right)=$ $\left.d\left(a_{1}, a_{6}\right)=2\right), d\left(a_{6}, a_{10}\right)=3$ (since $\left.d\left(a_{1}, a_{6}\right)=d\left(a_{1}, a_{10}\right)=2\right)$, and $d\left(a_{10}, a_{3}\right)=3$ (since $\left.d\left(a_{1}, a_{3}\right)=d\left(a_{1}, a_{10}\right)=2\right)$. This contradiction shows that Case I cannot apply to $M$.

Case II: $a_{4} \in N_{2}\left(a_{1}\right)$ and $a_{9} \notin N_{2}\left(a_{1}\right)$ : In this case we have $d\left(a_{1}, a_{5}\right)=3$ (or else $a_{1} a_{3} a_{5}$ would be equilateral), $d\left(a_{1}, a_{6}\right)=3$ (or else $a_{1} a_{4} a_{6}$ would be equilateral), and $d\left(a_{1}, a_{8}\right)=3$ (or else $a_{8} a_{10} a_{1}$ would be equilateral). This leaves us with $d\left(a_{1}, a_{7}\right)=2$, and we can now show that triangle $a_{4} a_{7} a_{10}$ is equilateral: in fact, $d\left(a_{4}, a_{7}\right)=3$ (or else $a_{1} a_{4} a_{7}$ would be equilateral), $d\left(a_{7}, a_{10}\right)=3$ (or else $a_{1} a_{7} a_{10}$ would be equilateral), and $d\left(a_{10}, a_{4}\right)=3$ (or else $a_{1} a_{4} a_{10}$ would be equilateral). This contradiction shows that Case II cannot apply to $M$, either, and so the Theorem is proved.

Theorem 15. The only maximal eq-free $\{1,2,4\}$-spaces (up to isomorphism) are $M_{2} \otimes$ $4 M_{1}$ and $M_{1} \otimes 2 M_{2}$ (see Example 7 for the definitions).

Proof. Let $M$ be a maximal (=10-point, by Theorem 11) eq-free $\{1,2,4\}$-space. By Theorem 2 and Lemma 8, the only types available for the elements of $M$ are

$$
[1,3,5], \quad[1,4,4], \quad[2,2,5], \quad[2,3,4], \quad[2,4,3]
$$

(as seen in the proof of Theorem 14, Lemma 8 implies that $\left|N_{1}\right| \neq \emptyset$ everywhere and Theorem 2 implies that the inequalities $\left|N_{2}\right| \leq 4$ and $\left|N_{4}\right| \leq 5$ must hold at every point, so the identity $\left|N_{1}\right|+\left|N_{2}\right|+\left|N_{4}\right|=9$ does the rest).

Case I: $M$ contains a point a of type $[*, *, 5]:\left|N_{4}(a)\right|=5$ means that $N_{4}(a)$ is isomorphic to $M_{2}$ (see Theorem 4 ), and so any point $b \in N_{4}(a)$ is necessarily of type $[2, *, *]$. If $N_{2}(b)$ contained three or more points, then one of these (call it $c$ ) would have to lie outside $N_{4}(a)$. Now, $d(c, b)=2$ implies that $d\left(c, b^{\prime}\right)=2$ for all five points in $N_{4}(a)$, and this because $d\left(c, b^{\prime}\right)$ cannot be $=1$, and by the triangle inequality can never be $=4$. However, this contradicts our choice of $M$, because there can never be more than 4 points at distance 2 from $c$ in $M$, or else there are equilateral triangles inside. It follows that 
$\left|N_{2}(b)\right|=2$, and so $b$ must be of type $[2,2,5]$. Now, $N_{4}(b) \sim M_{3}$ contains five points and is disjoint from $N_{4}(a) \sim M_{3}$, and so $M$ must be isomorphic to $M \sim M_{2} \otimes 4 M_{1}$.

Case II: $M$ only contains points of type $[2, *, *]$ : this is easily seen to lead to the same situation as in Case I. In fact, the points of $M$ could be then split into cycles of side 1 and of length at least 4 and at most 5 (since the internal distances within each cycle could only be 1 or 2 ). Since $|M|=10$, the only possibility is to have $M$ as a disjoint union of two cycles of length 5 , with all the distances between points belonging to different cycles being 4: and this means that $M \sim M_{2} \otimes 4 M_{1}$.

We thus have only one case left (but some more work to do):

Case III: $M$ contains a point of type $[1,4,4]$ (and the other points may only be of types $[2,3,4]$ or $[2,4,3])$ : Label the elements of $M$ as $a_{1}, \ldots, a_{10}$, and let $a_{1}$ be of type $[1,4,4]$. Let $a_{2}$ be the only point in $N_{1}\left(a_{1}\right)$, and choose the other labels so that $N_{2}\left(a_{1}\right)=$ $\left\{a_{3}, a_{4}, a_{5}, a_{6}\right\}$ and $N_{4}\left(a_{1}\right)=\left\{a_{7}, a_{8}, a_{9}, a_{10}\right\}$. Since $N_{2}\left(a_{1}\right)$ is a maximal $\{1,4\}$-space (see Theorems 2 and 4 ), we may set the distances within it as follows:

$$
d\left(a_{3}, a_{4}\right)=d\left(a_{5}, a_{6}\right)=1, \quad d\left(a_{3}, a_{5}\right)=d\left(a_{3}, a_{6}\right)=d\left(a_{4}, a_{5}\right)=d\left(a_{4}, a_{6}\right)=4 .
$$

Let us now consider $a_{3}$ (note that up to isomorphism this is an arbitrary point in $N_{2}\left(a_{1}\right)$ so far).

Claim 1: $a_{3}$ cannot be of type [2,3,4]. Suppose not, and think of $a_{3}$ as a type $[2,3,4]$ point. Given that $d\left(a_{1}, a_{3}\right)=2$ and that the four points in $N_{4}\left(a_{1}\right)$ must be at distance $\geq 2$ from $a_{3}$, the only other point in $N_{1}\left(a_{3}\right)$ must be $a_{2}$. Since $\left|N_{2}\left(a_{3}\right)\right|=3$, two of the points in $N_{4}\left(a_{1}\right)$ must be at distance 2 from $a_{3}$ : say, $a_{7}$ and $a_{8}$. Now, since $d\left(a_{2}, a_{3}\right)=1$ and $d\left(a_{3}, a_{7}\right)=2$, the triangle inequality implies that $d\left(a_{2}, a_{7}\right)=2$ ( $a_{2}$ has already been assigned two points at distance 1). However,

$$
4=d\left(a_{1}, a_{7}\right) \leq d\left(a_{1}, a_{2}\right)+d\left(a_{2}, a_{7}\right)=3,
$$

a contradiction proving Claim 1 .

Claim 2: $a_{3}$ cannot be of type $[2,4,3]$. This case proceeds in a similar way to the previous one: $a_{3}$ must be at distance 1 from $a_{2}$, and now three of the points in $N_{4}\left(a_{1}\right)$ must be at distance 2 from $a_{3}$ : just pick $a_{7}$ to be one of them and argue exactly as in the previous case to get another contradiction.

It thus follows from Claims 1 and 2 that $a_{3}$, and hence $a_{4}, a_{5}, a_{6}$, must all be of the only remaining type, that is, $[1,4,4]$. As a first consequence of this, we check that $D\left(a_{2}, a_{3}\right)=2$, and choose $a_{7}, a_{8}$ to be the two other points in $N_{2}\left(a_{3}\right)$. Since $N_{2}\left(a_{3}\right)$ is a maximal $\{1,4\}$-space, $d\left(a_{7}, a_{8}\right)=1$. We also have that $N_{4}\left(a_{3}\right)=\left\{a_{5}, a_{6}, a_{9}, a_{10}\right\}$ : now, this one is a $\{1,2\}$-space, and since $a_{5}, a_{6}$ are of type $[1,4,4]$, we must have

$$
d\left(a_{5}, a_{9}\right)=d\left(a_{5}, a_{10}\right)=d\left(a_{6}, a_{9}\right)=d\left(a_{6}, a_{10}\right) .
$$

Then, we note that $N_{4}\left(a_{3}\right)$ must now be $\left\{a_{5}, a_{6}, a_{9}, a_{10}\right\}$, which implies that $d\left(a_{9}, a_{10}\right)=1$ again by maximality of $N_{4}\left(a_{3}\right)$. Summarizing, it is now easy to get the following picture of $M$ : the pairs $S_{j}=\left\{a_{2 j-1}, a_{2 j}\right\}(j=1, \ldots, 5)$ are such that the distance between the two points within same the pair is 1 . Further, we can visualize the pairs in the order 
$S_{1}, S_{2}, S_{4}, S_{5}, S_{3}$ as sitting at the vertices of a pentagon: any two points from neighboring pairs are at distance 2, while points from "distant" pairs are at distance 4: and this is exactly the picture resulting from space $M_{1} \otimes 2 M_{2}$ (see Examples 6 and 7 ), as claimed.

Theorem 16. The only maximal eq-free $\{1,3,4\}$-space (up to isomorphism) is $M_{1} \otimes$ $\left(M_{2}+2\right)$ (see Example 7 for the definition). Also, a 9-point eq-free $\{1,3,4\}$-space must be isomorphic to a copy of $M_{1} \otimes\left(M_{2}+2\right)$ from which a point has been deleted.

Proof. Let $M$ be a maximal eq-free $\{1,3,4\}$-space. By Lemma 8 every point in $M$ must be at distance 1 from some other point, but since triangles with two sides of length 1 are not allowed here, the only available type for any point in $M$ is $[1,4,4]$ (in particular, every point has exactly one neighbor). It is now easy to derive the conclusion if we start thinking of $M$ as being built up of five pairs of points, where the distance within each pair is 1 , all other distances in $M$ being either 3 or 4 . For once we leave the details to the reader (the second part of the statement is also an easy exercise).

The following Lemmas contain much of the technicalities needed in the proof of Theorem 19 (we won't need their full power but we can't see the harm done by proving a stronger version):

Lemma 17. Let $n \geq 5$, and assume that a cycle $C_{n}$ (resp. a path $P_{n}$ ) belongs to an eq-free fms $M$. Label five consecutive points $b_{1}, \ldots, b_{5}$ on $C_{n}$ (resp. $P_{n}$ ) and assume that we have a sixth point $a \in M$ not adjacent to $b_{1}$, with $d\left(a, b_{2}\right)=d\left(a, b_{3}\right)=2$. Then we must have $d\left(a, b_{1}\right)=d\left(a, b_{4}\right)=3$ and $d\left(a, b_{5}\right)=4$.

Proof. $d\left(a, b_{5}\right)$ can only be 2,3 or 4 . It cannot be 2 , or else $a b_{3} b_{5}$ would be equilateral. On the other hand, $d\left(b_{1}, b_{3}\right)=2$ and so $d\left(b_{1}, b_{4}\right)=2$ (since $b_{1}$ and $b_{4}$ are both at distance 3 from $a$, and so they must be at a distance $\neq 3$ from each other $)$. If we had $d\left(b_{1}, b_{5}\right)=2$, then $b_{3}, b_{4}, b_{5}$ would be three consecutive points at distance 2 from $b_{1}$, and so they would form an equilateral triangle of side 1 . Consequently, $d\left(b_{1}, b_{5}\right)=3$, and if we had $d\left(a, b_{5}\right)=$ 3 the points $a b_{1} b_{5}$ would form an equilateral triangle of side 3 . Thus, $d\left(a, b_{5}\right)=4$, and the Lemma is proved.

Lemma 18. Suppose that $M$ is an eq-free fms.

(a) If $\left|N_{2}(a)\right| \geq 6$ for all $a \in M$, then $M$ contains no cycle $C_{4}$.

(b) If $\left|N_{2}(a)\right| \geq 8$ and $\left|N_{3}(a)\right| \geq 9$ for all $a \in M$, then $M$ contains no cycle $C_{m}$ with $m \geq 5\left(\left|N_{2}(a)\right| \geq 7\right.$ is enough for the case $\left.m=5\right)$.

(c) If $\left|N_{2}(a)\right| \geq 7$ and $\left|N_{3}(a)\right| \geq 9$ for all $a \in M$, then $M$ does not contain isomorphic copies of $P_{m}$ for any $m \geq 4$.

(d) If $\left|N_{1}(a)\right|=2$ for any fixed point in $M$, then $\left|N_{2}(a)\right| \leq 9$. 
Proof. (a): Suppose not, and let the four points around a $C_{4} \subset M$ be labelled $\left\{a_{1}, a_{2}\right.$, $\left.a_{3}, a_{4}\right\}$. Since

$$
\begin{aligned}
N_{2}\left(a_{2}\right) & =\left\{a_{4}\right\} \cup\left(N_{2}\left(a_{2}\right) \cap N_{2}\left(a_{1}\right)\right) \cup\left(N_{2}\left(a_{2}\right) \cap N_{3}\left(a_{1}\right)\right) \\
& =\left\{a_{4}\right\} \cup\left(N_{2}\left(a_{2}\right) \cap N_{2}\left(a_{3}\right)\right) \cup\left(N_{2}\left(a_{2}\right) \cap N_{3}\left(a_{3}\right)\right), \\
N_{2}\left(a_{4}\right) & =\left\{a_{2}\right\} \cup\left(N_{2}\left(a_{4}\right) \cap N_{2}\left(a_{1}\right)\right) \cup\left(N_{2}\left(a_{4}\right) \cap N_{3}\left(a_{1}\right)\right) \\
& =\left\{a_{2}\right\} \cup\left(N_{2}\left(a_{4}\right) \cap N_{2}\left(a_{3}\right)\right) \cup\left(N_{2}\left(a_{4}\right) \cap N_{3}\left(a_{3}\right)\right),
\end{aligned}
$$

and since the last set in all right hand side expressions contains at most 4 points (they are all $\{1,4\}$-spaces and Theorem 2 applies), it follows that the (disjoint) sets $A:=$ $N_{2}\left(a_{1}\right) \cap N_{2}\left(a_{4}\right)$ and $B:=N_{2}\left(a_{1}\right) \cap N_{2}\left(a_{2}\right)$ satisfy

$$
A \cup B \subset N_{2}\left(a_{1}\right) \cap N_{3}\left(a_{3}\right),
$$

so $A \cup B$ is an eq-free $\{1,4\}$-space. Now, looking at

$$
\begin{aligned}
N_{2}\left(a_{1}\right) & =\left\{a_{3}\right\} \cup\left(N_{2}\left(a_{1}\right) \cap N_{2}\left(a_{4}\right)\right) \cup\left(N_{2}\left(a_{1}\right) \cap N_{3}\left(a_{4}\right)\right) \\
& =\left\{a_{3}\right\} \cup\left(N_{2}\left(a_{1}\right) \cap N_{2}\left(a_{2}\right)\right) \cup\left(N_{2}\left(a_{1}\right) \cap N_{3}\left(a_{2}\right)\right),
\end{aligned}
$$

and defining $C:=N_{2}\left(a_{1}\right) \cap N_{3}\left(a_{4}\right) \cap N_{3}\left(a_{2}\right)$, we can write

$$
N_{2}\left(a_{1}\right)=\left\{a_{3}\right\} \cup A \cup B \cup C .
$$

We already know that $A \cup B$ is a $\{1,4\}$-space, and by (1) we also immediately see that $A \cup C$ and $B \cup C$ must also be $\{1,4\}$-spaces. However, this implies that $A \cup B \cup C$ is an eq-free $\{1,4\}$-space, and so $|A \cup B \cup C| \leq 4$, meaning that $\left|N_{2}\left(a_{1}\right)\right| \leq 5$.

(b): Suppose not, and let $C_{m} \subset M$. Let $m \geq 5$ and let $a_{1}, \ldots, a_{6}$ be six consecutive points on $C_{m}$ (omit $a_{6}$ in the case $m=5$ ). Since

$$
\begin{aligned}
N_{2}\left(a_{3}\right) & =\bigcup_{k=0}^{4} N_{2}\left(a_{3}\right) \cap N_{k}\left(a_{4}\right) \\
& =\left\{a_{5}\right\} \cup\left(N_{2}\left(a_{3}\right) \cap N_{2}\left(a_{4}\right)\right) \cup\left(N_{2}\left(a_{3}\right) \cap N_{3}\left(a_{4}\right)\right),
\end{aligned}
$$

since $\left|N_{2}\left(a_{3}\right) \cap N_{3}\left(a_{4}\right)\right| \leq 4$ (it's a $\{1,4\}$-space), and since by hypothesis $\left|N_{2}\left(a_{3}\right)\right| \geq 8$, we must have at least one point at distance 2 from both $a_{3}$ and $a_{4}$, and different from both $a_{1}$ and $a_{6}$ (we only need $\left|N_{2}\left(a_{3}\right)\right| \geq 7$ in the case $m=5$, as we just want to pick a point different from $\left.a_{1}\right)$. Call such a point $b$ : by Lemma 17 we must have $d\left(b, a_{2}\right)=d\left(b, a_{5}\right)=3$, and (if $m>5) d\left(b, a_{1}\right)=d\left(b, a_{6}\right)=4$, that is, $N_{3}(b)$ contains two singletons if $m>5$ and either two singletons or three points forming a $P_{3}$-subspace of $N_{3}\left(a_{3}\right)$ if $m=5$ : so, $N_{3}(b)$ could not contain 9 points or more, by Theorem 15 .

(c): Suppose not, and let $P_{m} \subset M$, with $m \geq 4$ and the points along $P_{m}$ being labelled as $\left\{a_{1}, \ldots, a_{m}\right\}$. Since

$$
\begin{aligned}
N_{2}\left(a_{2}\right) & =\bigcup_{k=0}^{4} N_{2}\left(a_{2}\right) \cap N_{k}\left(a_{3}\right) \\
& =\left\{a_{4}\right\} \cup\left(N_{2}\left(a_{2}\right) \cap N_{2}\left(a_{3}\right)\right) \cup\left(N_{2}\left(a_{2}\right) \cap N_{3}\left(a_{3}\right)\right),
\end{aligned}
$$

THE ELECTRONIC JOURNAL OF COMBINATORICS 11 (2004), \#R18 
and since $\left|N_{2}\left(a_{2}\right) \cap N_{3}\left(a_{3}\right)\right| \leq 4$ (it's a $\{1,4\}$-space, and so Theorem 2 applies), and since the hypothesis implies that $\left|N_{2}\left(a_{2}\right)\right| \geq 7$, we must have at least two points at distance 2 from both $a_{2}$ and $a_{3}$. Pick one of them that is $\neq a_{5}$ and call it $b$. Since no three consecutive points in $P_{m}$ can be at distance 2 from $b$ (or else they would need to form an equilateral triangle of side 1$)$, we must have $d\left(b, a_{1}\right)=d\left(b, a_{4}\right)=3$ (since $b \neq a_{5}$ we cannot have $\left.d\left(b, a_{4}\right)=1\right)$. Now, if $m=4$ we have that $P_{4}=\left\{a_{1}, \ldots, a_{4}\right\}$ contains two singletons from $N_{3}(b)$, which is incompatible with the hypothesis that $\left|N_{3}(b)\right| \geq 9$. If instead $m>4$, we can look at $d\left(b, a_{5}\right)$ and conclude that it must be $=4$ by Lemma 17 , which again gives us two singletons in $N_{3}(b)$.

(d): Let $N_{1}(a)=\{b, c\}$, and assume by contradiction that $\left|N_{2}(a)\right|=10$. Writing

$$
\begin{aligned}
N_{2}(a) & =\left(N_{2}(a) \cap N_{1}(b)\right) \cup\left(N_{2}(a) \cap N_{2}(b)\right) \cup\left(N_{2}(a) \cap N_{3}(b)\right) \\
& =\left(N_{2}(a) \cap N_{1}(c)\right) \cup\left(N_{2}(a) \cap N_{2}(c)\right) \cup\left(N_{2}(a) \cap N_{3}(c)\right),
\end{aligned}
$$

we note that the first set on both right hand sides contains at most one point, and the third set contains at most four points. Since $\left|N_{2}(a)\right|=10$, the second set must contain at least five points. However, since $N_{2}(a) \cap N_{2}(b)$ and $N_{2}(a) \cap N_{2}(c)$ are disjoint (due to $d(b, c)=2$, and thus $\left.N_{2}(b) \cap N_{2}(c)=\emptyset\right)$, they must both contain exactly five points. We can deduce quite a bit from this. First, $\left|N_{2}(a) \cap N_{1}(b)\right|=\left|N_{2}(a) \cap N_{1}(c)\right|=1$, and so there exist points $e, f$ with $N_{2}(a) \cap N_{1}(b)=\{e\}$ and $N_{2}(a) \cap N_{1}(c)=\{f\}$. Note that $d$ and $e$ must be different, or else the five point set $N_{2}(a) \cap N_{2}(b)$ would need to be contained in the four point set $N_{2}(a) \cap N_{3}(c)$. With this notation we have

$$
A:=\left(N_{2}(a) \cap N_{2}(b)\right) \backslash\{f\}=N_{2}(a) \cap N_{3}(c)
$$

and

$$
B:=\left(N_{2}(a) \cap N_{2}(c)\right) \backslash\{e\}=N_{2}(a) \cap N_{3}(b) .
$$

Both $A$ and $B$ contain four points and thus are maximal $\{1,4\}$-spaces, for which the isomorphic structure is uniquely defined (i.e., in both $A$ and $B$ we find two pairs of neighbors, with points from different pairs being at distance 4 ). Since we have

$$
N_{2}(a)=A \cup B \cup\{e, f\},
$$

applying Theorem 16 we see that we must have $d(e, f)=1$ (which means that the points $a, b, c, e, f$ form an isomorphic copy of $M_{2}$ inside $M$ ). On the other hand, since all the points in $A$ are at distance 2 from both $a$ and $b$, they all must be at distance 3 from $e$. Given the unique isomorphic structure of $N_{2}(a)$, this gives a contradiction, since two of the points of $A$ must be at distance 4 from $e$.

We are now in a position to identify the value of $D_{4}$ :

Theorem 19. $D_{4}=D[1,2,3,4]=33$. We also have $D[1,2,3,5]=D[1,2,4,5]=26$. 
Proof. $D_{4}=D[1,2,3,4]=33$ : In [5] (see also [6] and [8]), Greenwood and Gleason were the first to prove that there exists a 3-coloring of the edges of a complete graph with 16 points such that no triangle has all the edges of the same color. Pick these 16 points and this coloring, and instead of the three colors "paint" the edges with the distances 2, 3 and 4 (the triangle inequality doesn't impose any restrictions here). Now, consider every point among the 16 to really be a pair of points at distance 1 from each other, while defining the distances between points belonging to different pairs according to the same $\{2,3,4\}$-color scheme used at the start. The resulting configuration is easily seen to be a 32 -point eq-free $\{1,2,3,4\}$-space, thus establishing that $D_{4} \geq 33$.

Let us now prove that $D_{4} \leq 33$. By contradiction, assume that $M$ is a 33-point eq-free $\{1,2,3,4\}$-space. Given the bounds in Theorems 2 and 11, and part (d) of Lemma 18 (i.e., the inequalities $\left|N_{2}\right| \leq 10,\left|N_{3}\right| \leq 10$ and $\left|N_{4}\right| \leq 11$ must hold everywhere), the only types allowed for the points in $M$ are

$$
[1,10,10,11], \quad[2,9,10,11] .
$$

If a point $a$ were of type $[*, *, *, 11]$, though, $M$ would have to contain a copy of $M_{3}$ $\left(=N_{4}(a)\right)$, and by Theorem $13|M| \leq 22$, a contradiction.

$D[1,2,3,5]=26$ : The $\{1,2,3,5\}$-space $M_{2} \otimes\left(2 M_{2}+1\right)$ is eq-free and contains 25 points, and so $D[1,2,3,5] \geq 26$ (drawing a sketch of $M_{2} \otimes\left(2 M_{2}+1\right)$ will make things easier here: it looks like five copies of $M_{2}$ arranged at the vertices of a bigger pentagon with "sides" of length 3 and "diagonals" of length 5: verification that this space is indeed metric and eq-free is then trivial).

Let us assume by contradiction that $M$ is a 26-point eq-free $\{1,2,3,5\}$-space. Since for any $a \in M$ we have that $N_{2}(a)$ is a $\{1,3\}$-space, it follows that $\left|N_{2}(a)\right| \leq 4$ by Theorem 2. Similarly, Theorem 11 implies that $\left|N_{3}(a)\right| \leq 10$ and $\left|N_{5}(a)\right| \leq 11$. If $\left|N_{5}(a)\right|=11$ for some $a \in M$, then by Theorem $14 N_{5}(a)$ would be isomorphically determined as a copy of $M_{3}$ and, in particular, it would be a cycle $C_{11}$. By the triangle inequality, any $b \in M \backslash N_{5}(a)$ would have to be either at distance 2 or 3 from all points of $N_{5}(a)$, or else $N_{5}(b)=N_{5}(a)$. Since the first possibility is plainly absurd, we deduce that if $b \notin N_{5}(a)$ we have $d(a, b) \leq 3$, and so $M \backslash N_{5}(a)$ would be a $\{1,2,3\}$-space: but by Theorem 11 this would imply $|M| \leq 22$, a contradiction. So, $\left|N_{5}(a)\right| \leq 10$ for all $a \in M$.

From this, it follows that if $\left|N_{1}(a)\right|=1$, then $a$ must be of type $[1,4,10,10] .\left|N_{3}(a)\right|=$ 10 says that $N_{3}(a)$ is a maximal eq-free $\{1,2,5\}$-space, and it is easy to see that the only isomorphic shape for $N_{3}(a)$ is thus $M_{2} \otimes 5 M_{1}$ : so, $M$ must contain a copy of $M_{2}$ (i.e., of a cycle $C_{5}$ : let's just call this copy $C_{5}$, for simplicity). Now, if $b$ is a point in $M \backslash C_{5}$ at distance 2 from some point of $C_{5}$ (and there is such a point, since $\left|N_{2}(c)\right| \geq 3$ for all $c \in M$ as a consequence of $|M|=26$ ), it is easy to derive a contradiction: either $b$ is of type $[1,4,10,10]$ (in which case $b$ must be at distance 2 from two neighbors in $C_{5}$, and at distance 3 from the others three points of $C_{5}$, contradicting the mandatory shape of $\left.N_{3}(b)\right)$, or else $b$ is of one of the types $[2,3,10,10],[2,4,9,10],[2,4,10,9]$, and then in any of these cases we derive a similar contradiction.

Consequently, we must have $\left|N_{1}(a)\right|=2$ for all $a$, and so $M$ breaks down into a disjoint union of cycles. Since the argument we just went through shows that no $C_{5}$ can 
be a subspace of $M$, it must be that all the points in $M$ are of type $[2,4,9,10]$. For any $a \in M, N_{2}(a)$ being then a maximal four-point $\{1,3\}$-space, it must consist of two pairs of neighbors, and this easily implies that the cycles in $M$ must have at least 9 points each, and so $N_{5}(a)$ is always a cycle $C_{10}$ for every $a \in M$. Now, this clearly implies that $M$ needs to contain two copies of $C_{10}$, but then the remaining six points cannot for a cycle with nine points or more: a contradiction.

$D[1,2,4,5]=26$ : The $\{1,2,4,5\}$-space $M_{2} \otimes\left(M_{2}+3\right)$ is eq-free and contains 25 points, and so $D[1,2,4,5] \geq 26$ (as in the previous part of this proof, the reader is advised to make a sketch of $M_{2} \otimes\left(M_{2}+3\right)$ : it looks like five copies of $M_{2}$ arranged at the vertices of a bigger pentagon with "sides" of length 4 and "diagonals" of length 5: verification that this space is indeed metric and eq-free is then trivial).

Let us assume by contradiction that $M$ is a 26 -point eq-free $\{1,2,4,5\}$-space. Since for any $a \in M$ we have that $N_{2}(a)$ is a $\{1,4\}$-space, it follows that $\left|N_{2}(a)\right| \leq 4$ by Theorem 2 . Similarly, Theorem 11 implies that $\left|N_{4}(a)\right| \leq 10$ and $\left|N_{5}(a)\right| \leq 10$. So, the only available types in $M$ are $[1,4,10,10],[2,3,10,10],[2,4,9,10]$, or $[2,4,10,9]$.

Since the triangle inequality implies that the distances within any cycle or path in $M$ can only be 1 or 2 , we immediately deduce that $M$ is a disjoint union of copies of $P_{2}, P_{3}, P_{4}, C_{4}$ or $C_{5} . C_{5}$ is impossible for the same reason seen in the previous case. $P_{3}$ is impossible because one endpoint of $P_{3}$ would need to be of type $[*, 4, *, *]$, and yet it would be at distance 2 from the other endpoint, a contradiction with the unique isomorphic shape that is possible for $N_{2}(a)$ (two pairs of neighbors). $P_{4}$ is impossible for the following reason: let $a_{1}, a_{2}, a_{3}, a_{4}$ be the four consecutive points in $P_{4}$. Then

$$
\begin{aligned}
N_{2}\left(a_{2}\right) & =N_{2}\left(a_{2}\right) \cap N_{2}\left(a_{1}\right) \\
& =\left\{a_{4}\right\} \cup\left(N_{2}\left(a_{2}\right) \cap N_{2}\left(a_{3}\right)\right)
\end{aligned}
$$

and, since $N_{2}\left(a_{1}\right)$ and $N_{2}\left(a_{3}\right)$ are disjoint, we deduce the contradiction $\left|N_{2}\left(a_{2}\right)\right|=1$. A totally similar argument also shows that $C_{4}$ cannot be a subspace of $M$, which leaves us with $M$ being made up of 13 copies of $P_{2}$, and thus every point in $M$ is of type $[1,4,10,10]$. However, since now (for any $a \in M) N_{4}(a)$ is a maximal eq-free $\{1,2,5\}$-space, $M$ is forced to contain copies of $C_{5}$, which leads to a contradiction because we have already seen that there is no place for $C_{5}$ in $M$.

Theorem 20. There exist exactly two non-isomorphic (maximal) eq-free $\{1,2,3,4\}$-spaces with 32 points.

Proof. Assume that $M$ is a 32 -point eq-free $\{1,2,3,4\}$-space. Given the bounds in Theorems 2 and 11, the fact that (by Theorem 13) no point can be of type $[*, *, *, 11]$, and type $[2,10, *, *]$ being outlawed by Lemma 18 , the only types allowed for the points in $M$ are

$$
[1,10,10,10] \text { and }[2,9,10,10] \text {. }
$$

With this information in hand we can quickly zoom into the structure of $M$ :

(A) $M$ contains no cycles $C_{n}$ (with $n \geq 3$ ). This follows immediately from Lemma 18 . 
(B) $M$ contains no path $P_{m}$ with $m \geq 3$. The statement for $m \geq 4$ is a corollary of Lemma 18. If $P_{3}=\left\{a_{1}, a_{2}, a_{3}\right\}$ were a subset of $M$, we would see that $a_{3}$ would be a singleton inside $N_{2}\left(a_{1}\right)$, but this would contradict the structure spelled out in Theorem 16 since $a_{1}$ must be of type $[1,10,10,10]$, and thus $N_{2}\left(a_{1}\right)$ must be a maximal eq-free $\{1,3,4\}$-space.

It thus follows that $M$ breaks down into 16 copies of $P_{2}$, and it is immediate to see that under these circumstances the two points belonging to the same $P_{2}$ must share the same distance from any other point in $M$. So, we could "collapse" each $P_{2}$ into a single element of a 16-point eq-free $\{2,3,4\}$-space: the resulting 16 -point space can thus be considered a complete graph whose edges have been colored with the colors 2,3 and 4, and such that no triangles have all sides of the same color. By the result of Kalbfleisch and Stanton [6] (see also [8]) there are (up to isomorphism) exactly two ways to achieve this, each of these two ways produces an isomorphically different maximal eq-free $\{1,2,3,4\}$-space.

We conclude this paper with some inequalities that form a prelude for the study of the asymptotics of the numbers $D_{n}$ (which we hope will be the main topic of a future paper, together with the analysis of the relationship with the so-called Schur numbers).

Lemma 12 is the special case $m=1$ of the following Theorem:

Theorem 21. Suppose that $M$ is a maximal eq-free $\{1, \ldots, m\}$-space, and that $N$ is a maximal eq-free $\{1, \ldots, n\}$-space. Then $M \otimes(N+m)$ is an eq-free $\{1, \ldots, m+n\}$-space (see Example 7 for a definition of $\otimes$ ). In particular, we have the inequality

$$
D_{m+n} \geq\left(D_{m}-1\right)\left(D_{n}-1\right)+1 \text {. }
$$

Proof. $M$ and $N$ being as in the statement, $E:=M \otimes(N+m)$ clearly has distances in the set $\{1, \ldots, m+n\}$. We need to prove that it is an eq-free $\{1, \ldots, m+n\}$-space. We can think of $E$ as $N$, where (1) every distance has been increased by $m$; (2) every point has been replaced by an isometric copy of $M$; and (3) the distance between two points belonging to different copies of $M$ is the same as the one between the two original points from $N+n$. So, if we pick three points from $E$ we distinguish between three cases:

(A) the three points belong to the same copy of $M$ : then the triangle cannot be equilateral, since $M$ is assumed to be eq-free. Also, the triangle is metric because it lives inside the original metric of $M$.

(B) two of the points (say, $a$ and $b$ ) belong to the same copy of $M$, the third (say, $c$ ) belongs to a different one: in this case $d(a, b) \leq m$ since $M$ is a $\{1, \ldots, m\}$-space, while $d(a, c)=d(b, c) \in\{m+1, \ldots, m+n\}$, and so $a b c$ is not equilateral. As for the triangle being metric, since $d(a, c)=d(b, c)$ are the longer sides, we have no problem here, either.

(C) the three points belong to three different copies of $M$ : in this case the distances are governed by the distance table of $N+n$, and so we cannot have an equilateral triangle since $N$ was assumed to be eq-free. The triangle is metric in this case, 
too, since the three sides have the lengths of an original triangle inside $N$, each augmented by $m$, which only makes the triangle inequality even easier to satisfy.

$E$ is thus shown to be an eq-free $\{1, \ldots, m+n\}$-space, and so we must have $D_{m+n} \geq$ $|E|+1$ : the inequality follows easily.

Theorem 22. For all $n \geq 4$ we have the inequality

$$
D_{n} \geq 1+\left(D_{\left\lceil\frac{n}{2}\right\rceil-1}-1\right)\left(R_{\left\lfloor\frac{n}{2}\right\rfloor+1}-1\right) .
$$

We have $D_{5} \leq 95$ and, for all $n \geq 6$,

$$
D_{n} \leq 15+\lceil n / 2\rceil D_{n-1}+\left(\sum_{k=3}^{\lfloor n / 2\rfloor} D_{2 k-1}\right)-n .
$$

Proof. Inequality (2) is checked as follows: first consider an eq-free $\left\{\left\lceil\frac{n}{2}\right\rceil, \ldots, n\right\}$-space $E$ with $R_{\left\lfloor\frac{n}{2}\right\rfloor+1}-1$ points (we used $n-\left\lceil\frac{n}{2}\right\rceil=\left\lfloor\frac{n}{2}\right\rfloor$ ): this is achieved by simply considering the distances as colors, and noting that the triangle inequality doesn't impose any restrictions for this particular range of distances. On the other hand, let $F$ be an eq-free $\left\{1, \ldots,\left\lceil\frac{n}{2}\right\rceil-\right.$ $1\}$-space with $D_{\left\lceil\frac{n}{2}\right\rceil-1}-1$ points: the space $F \otimes E$ is easily seen to be an eq-free $\{1, \ldots, n\}$ space with $\left(D_{\left\lceil\frac{n}{2}\right\rceil-1}-1\right)\left(R_{\left\lfloor\frac{n}{2}\right\rfloor+1}-1\right)$ points, proving $(2)$.

To prove inequality (3) for $n \geq 5$, we consider a maximal eq-free $\{1, \ldots, n\}$-space $M$, and for a fixed $a \in M$ we use the obvious $|M|=1+\sum_{k=1}^{n}\left|N_{k}(a)\right|$ and estimate each $\left|N_{k}(a)\right|$ as follows: Lemma 18 implies that we must always have $\left|N_{1}(a)\right|+\left|N_{2}(a)\right| \leq 11$. Next, for $3 \leq k \leq\lfloor n / 2\rfloor$, we have

$$
\left|N_{k}(a)\right| \leq D[1, \ldots, k-1, k+1, \ldots, 2 k] \leq D_{2 k-1}
$$

(because two points at distance $k$ from $a$ cannot be at distance more than $2 k$ from each other).

If $k>\lfloor n / 2\rfloor$ we use the estimate

$$
\left|N_{k}(a)\right| \leq D[1, \ldots, k-1, k+1, \ldots, n] \leq D_{n-1} .
$$

This easily yields the inequality (3). If $n=5$, we can improve on the above argument because if $a \in M$ is fixed, we have that $N_{3}(a)$ is a $\{1,2,4,5\}$-space, and $N_{4}(a)$ is a $\{1,2,3,5\}$-space: Theorem 19 thus implies that $\left|N_{3}(a)\right|$ and $\left|N_{4}(a)\right|$ can never be greater that 25 . So, $|M|$ can in this case be estimated by the sum $1+2+9+25+25+32=94$.

Corollary 23. We have

$$
81 \leq D_{5} \leq 95, \quad 251 \leq D_{6} \leq 389, \quad 551 \leq D_{7} \leq 1659 .
$$

Proof. The lower estimates are a corollary of Theorem 22, while using the known results $R_{3}=17$ and $R_{4} \geq 51$. The upper estimates follow readily from Theorem 22 . 
Defining the sequence $d_{3}:=12, d_{4}:=33, d_{5}:=95$ and (for $n \geq 6$ )

$$
d_{n}:=(\lfloor n / 2\rfloor+2) d_{n-1}-\lfloor n / 2\rfloor d_{n-2}-1,
$$

we obtain a faster way to calculate the same estimates for $D_{n}$ :

$$
D_{n} \leq d_{n}
$$

It's a standard calculus exercise to show that this implies the rough estimate $D_{n} \leq$ $c(n+4) ! / 2^{n}$ (we leave the verification as an exercise, though we have serious doubts about the usefulness of such sub-optimal estimates).

Finally, let us recall that the $\operatorname{limit}_{n \rightarrow \infty} R_{n}^{1 / n}$ exists (but not much more is known except for this limit lying in the interval $(3.19, \infty]$ : see [2] for an early exposition, and the recent paper [4] for what's needed to prove the lower bound). It is easy to deduce from Theorems 21 and 22 (and the trivial inequality $\left.D_{n} \leq R_{n}\right)$ that the sequence $\left(D_{n}^{1 / n}\right)$ converges, and

$$
\lim _{n \rightarrow \infty} D_{n}^{1 / n}=\lim _{n \rightarrow \infty} R_{n}^{1 / n} .
$$

So, any progress made on the asymptotics of the $D_{n}$ numbers should be shedding light on the classical $R_{n}$ numbers as well. As already stated, the author is planning a further study focusing on these issues.

\section{References}

[1] F.R.K. Chung: On the Ramsey numbers $N(3,3, \ldots, 3 ; 2)$, Discrete Mathematics $\mathbf{5}$ (1973) 317-21.

[2] F.R.K. Chung, C.M. Grinstead: A survey of bounds for classical Ramsey numbers, Journal of Graph Theory 7 (1983) 25-37.

[3] S. Fettes, R.L. Kramer, S. Radziszowski: An upper bound of 62 on the classical Ramsey number $R(3,3,3,3)$, to appear in Ars Combinatoria.

[4] H. Fredricksen, M. M. Sweet: Symmetric sum-free partitions and lower bounds for Schur numbers, Electronic J. Combin. 7 (2000), R32, 9pp.

[5] R. E. Greenwood, A. M. Gleason: Combinatorial relations and chromatic graphs, Canad. J. Math. 7 (1955) 1-7.

[6] J. G. Kalbfleisch, R. G. Stanton: On the maximal triangle-free edge-chromatic graphs in three colors, J. Combin. Theory 5 (1968) 9-20.

[7] R.L. Kramer: The classical Ramsey number $R(3,3,3,3 ; 2)$ is no greater than 62, unpublished manuscript (available for download as a .pdf file at the website http://www . math. iastate. edu/maddux/papers/ram.pdf). 
[8] C. Laywine, J. P. Mayberry: A simple construction giving the two non-isomorphic triangle-free 3 colored $K_{16}$ 's, J. Combin. Theory, Series B 45 (1988) 120-4.

[9] S.P. Radziszowski: Small Ramsey Numbers, Electronic J. Combin. (2002), DS1.9 (dynamic survey, revision \# 9 dated July 15, 2002), 42pp.

[10] F.P. Ramsey: On a problem of formal logic, Proc. London Math. Soc. (2) 30 (1930) 264-286. 